\title{
Recent Developments in Gauge Theories
}




\section{NATO ADVANCED STUDY INSTITUTES SERIES}

A series of edited volumes comprising multifaceted studies of contemporary scientific issues by some of the best scientific minds in the world, assembled in cooperation with NATO Scientific Affairs Division.

\section{Series B. Physics}

\section{Recent Volumes in this Series}

Volume 52 - Physics of Nonlinear Transport in Semiconductors edited by David K. Ferry, J. R. Barker, and C. Jacoboni

Volume 53 - Atomic and Molecular Processes in Controlled Thermonuclear Fusion edited by M. R. C. McDowell and A. M. Ferendeci

Volume 54 - Quantum Flavordynamics, Quantum Chromodynamics, and Unified Theories edited by K. T. Mahanthappa and James Randa

Volume 55 - Field Theoretical Methods in Particle Physics edited by Werner Rühl

Volume 56 - Vibrational Spectroscopy of Molecular Liquids and Solids edited by S. Bratos and R. M. Pick

Volume 57 - Quantum Dynamics of Molecules: The New Experimental Challenge to Theorists edited by R. G. Woolley

Volume 58 - Cosmology and Gravitation: Spin, Torsion, Rotation, and Supergravity edited by Peter G. Bergmann and Venzo De Sabbata

Volume 59 - Recent Developments in Gauge Theories edited by G. 't Hooft, C. Itzykson, A. Jaffe, H. Lehmann,

P. K. Mitter, I. M. Singer, and R. Stora

Volume 60 - Theoretical Aspects and New Developments in Magneto-Optics Edited by Jozef T. Devreese

Volume 61 - Quarks and Leptons: Cargèse 1979 edited by Maurice Lévy, Jean-Louis Basdevant, David Speiser, Jacques Weyers, Raymond Gastmans, and Maurice Jacob

Volume 62 - Radiationless Processes edited by Baldassare Di Bartolo

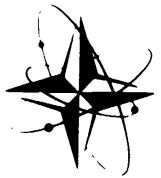

This series is published by an international board of publishers in conjunction with NATO Scientific Affairs Division
A Life Sciences
B Physics
C Mathematical and Physical Sciences
D Behavioral and Social Sciences
E Applied Sciences

Plenum Publishing Corporation
D. Reidel Publishing Company Dordrecht, Boston and London

Sijthoff \& Noordhoff International Publishers

Alphen aan den Rijn, The Netherlands, and Germantown U.S.A. 


\section{Recent Developments in Gauge Theories}

Edited by

G. 't Hooft

Institute for Theoretical Physics

Utrecht, The Netherlands

C. Itzykson

CEN Saclay

Gif-sur-Yvette, France

A. Jaffe

Harvard University

Cambridge, Massachusetts

H. Lehmann

University of Hamburg

Hamburg, Federal Republic of Germany

P. K. Mitter

University of Paris

Paris, France

\section{M. Singer}

University of California

Berkeley, California

and

R. Stora

Center of Theoretical Physics, CNRS

Marseille, France

\section{PLENUM PRESS • NEW YORK AND LONDON}

Published in cooperation with NATO Scientific Affairs Division 


\section{Library of Congress Cataloging in Publication Data}

Main entry under title:

Recent developments in gauge theories.

(NATO advanced study institutes series: Series B, Physics; v. 59)

"Published in cooperation with NATO Scientific Affairs Division."

Includes index.

1. Gauge fields (Physics)-Addresses, essays, lectures. I. 't Hooft, G. II. Series.

$530.1^{\prime} 43$

80-18528

ISBN 978-1-4684-7573-9 ISBN 978-1-4684-7571-5 (eBook)

DOI $10.1007 / 978-1-4684-7571-5$

Proceedings of the NATO Advanced Study Institute on

Recent Developments in Gauge Theories, held in Cargèse, Corsica,

August 26-September 8, 1979.

(c) 1980 Plenum Press, New York

Softcover reprint of the hardcover 1st edition 1980

A Division of Plenum Publishing Corporation

227 West 17th Street, New York, N.Y. 10011

All rights reserved

No part of this book may be reproduced, stored in a retrieval system, or transmitted, in any form or by any means, electronic, mechanical, photocopying, microfilming, recording, or otherwise, without written permission from the Publisher 


\section{PREFACE}

Almost all theories of fundamental interactions are nowadays based on the gauge concept. Starting with the historical example of quantum electrodynamics, we have been led to the successful unified gauge theory of weak and electromagnetic interactions, and finally to a non abelian gauge theory of strong interactions with the notion of permanently confined quarks. The early theoretical work on gauge theories was devoted to proofs of renormalizability, investigation of short distance behaviour, the discovery of asymptotic freedom, etc.., aspects which were accessible to tools extrapolated from renormalised perturbation theory. The second phase of the subject is concerned with the problem of quark confinement which necessitates a non-perturbative understanding of gauge theories. This phase has so far been marked by the introduction of ideas from geometry, topology and statistical mechanics in particular the theory of phase transitions. The 1979 Cargèse Institute on "Recent Developments on Gauge Theories" was devoted to a thorough discussion of these non-perturbative, global aspects of non-abelian gauge theories. In the lectures and seminars reproduced in this volume the reader will find detailed reports on most of the important developments of recent times on non perturbative gauge fields by some of the leading experts and innovators in this field. Aside from lectures on gauge fields proper, there were lectures on gauge field concepts in condensed matter physics and lectures by mathematicians on global aspects of the calculus of variations, its relation to geometry and topology, and related topics. The presence of mathematicians as enthusiastic participants and masterful lecturers in this school deserves special mention. We hope this trend will continue in the future and that, in the 1 ast quarter of this century, common concerns about the fundamental interactions will bring ever closer the physical and mathematical communities as in the days of yore.

We wish to express our gratitude to NATO whose generous financial contribution made it possible to organise this school. We also thank the Centre National de la Recherche Scientifique, the Délégation à la Recherche Scientifique et Technique, the 
C.E.N. de Saclay, as well as the University of Hamburg for financial help. We thank the University of Nice for making available to us the facilities of the Institut d'Etudes Scientifiques de Cargèse. Grateful thanks are due to Marie-France Hanseler for much help with the material aspects of the organisation. Last but not least we thank the lecturers and participants for their enthusiastic involvement which contributed much to the scientific atmosphere of the school.

\author{
G. ' $t$ Hooft \\ C. Itzykson \\ A. Jaffe \\ H. Lehmann \\ P.K. Mitter \\ I.M. Singer \\ R. Stora
}


CONTENTS

Remarks on Morse Theory . . . . . . . . . . . . 1

M.F. Atiyah

Morse Theoretic Aspects of Yang-Mills Theory . . . . . . 7

R. Bott

A Semiclassical Approach to the Strong Coupling

Physics of QCD . . . . . . . . . . . . .

C.G. Callan, Jr.

Lattice Gauge Theories . . . . . . . . . . . .

J. Glimm

Some Results and Comments on Quantized Gauge

Fields . . . . . . . . . . . . . . .

J. Fröh1ich

String States in Q.C.D. . . . . . . . . . . . . .

J.-L. Gervais and A. Neveu

Why Do We Need Local Gauge Invariance in

Theories with Vector Particles?

An Introduction. (Lecture I) ...........

G. 't Hooft

Which Topological Features of a Gauge Theory

can be Responsible for Permanent

Confinement? (Lecture II) . . . . . . . . . .

G.'t Hooft

Naturalness, Chiral Symmetry, and Spontaneous

Chiral Symmetry Breaking. (Lecture III) . . . .

G. 't Hooft

Introduction to Lattice Gauge Theories . . . . . . .

C. Itzykson 
Classical Gauge Theories and Their Quantum

A. Jaffe

The Coupling Constant in a $\emptyset^{4}$ Field Theory . . . . . .

J. Glimm and A. Jaffe

Exact Instanton Gases . . . . . . . . . . . .

M. Lüscher

Properties of Lattice Gauge Theory Models at Low Temperatures . . . . . . . . . .

G. Mack

Geometry of the Space of Gauge Orbits and the

Yang-Mills Dynamical System . . . . . . . . P.K. Mitter

On the Structure of the Phases in Lattice Gauge Theories . . . . . . . . . . . .

G. Parisi

Superalgebras and Confinement in Condensed

Matter Physics . . . . . . . . . . .

V. Poénaru

Cut off Dependence in Lattice $\phi^{4}$ Theory . . . . . . .
K. Symanzik

Gauge Concepts in Condensed Matter Physics . . . .

G. Toulouse

Monte-Carlo Calculations for the Lattice

Gauge Theory . . . . . . . . . . . .

K.G. Wilson

The 1/N Expansion in Atomic and Particle Physics . . . . . . . . . . . . . . .

E. Witten

Generalized Non-Linear $\sigma$-Mode1s with Gauge

Invariance

J. Zinn-Just in

Index . . . . . . . . . . . . . . . . . 